
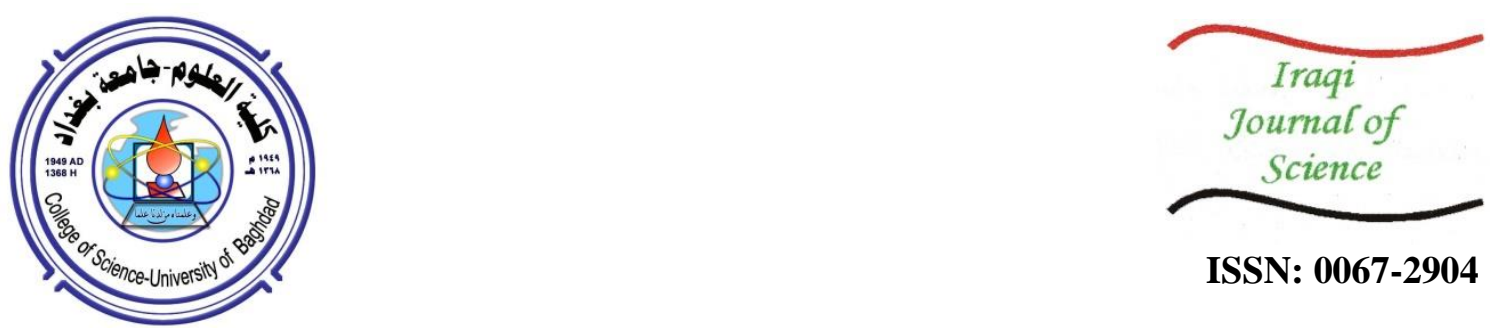

ISSN: 0067-2904

\title{
Symmetry Group for Solving Elliptic Euler-Poisson-Darboux Equation
}

\author{
Zainab John
}

Department of Mathematics, College of Science, Al-Mustansiriyah University, Iraq

Received: $14 / 11 / 2019 \quad$ Accepted: $3 / 4 / 2020$

\begin{abstract}
:
The aim of this article is to study the solution of Elliptic Euler-Poisson-Darboux equation, by using the symmetry of Lie Algebra of orders two and three, as a contribution in partial differential equations and their solutions.
\end{abstract}

Keywords: symmetry, Ellipic Euler-poisson-Darboux, Lie Algebra
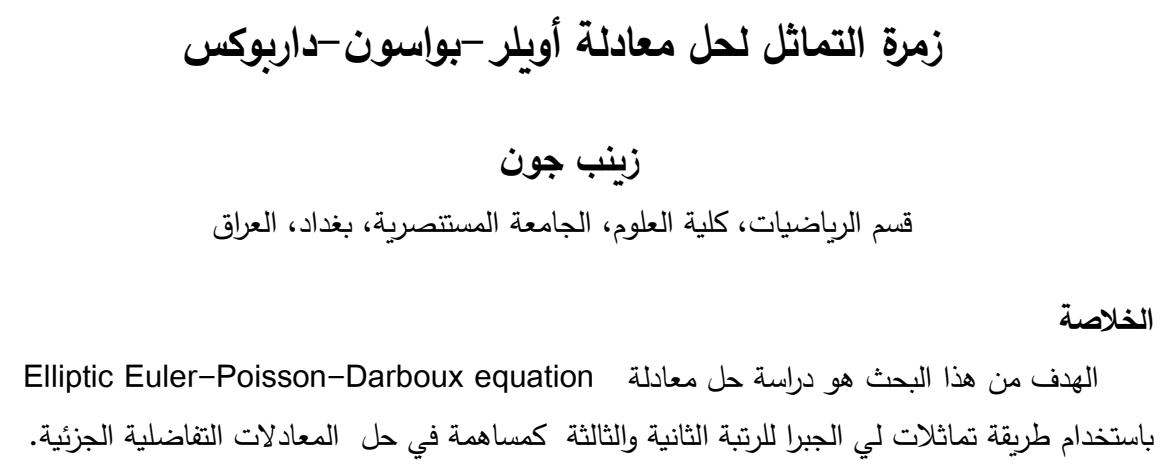

\section{Introduction}

The Euler-Poisson-Darboux equation is very important in physics and mathematics. It is one of the most extensively studied singular linear hyperbolic equations. The general formula of Euler- PoissonDarboux Equation $([1,2,3]$ is:

$$
\frac{\partial^{2} u}{\partial r^{2}}+\frac{\alpha}{r} \frac{\partial u}{\partial r}=\mp \sum_{i=1}^{n} \frac{\partial^{2} u}{\partial z_{i}^{2}} u=(r, z ; \alpha), r \in R^{n}, \mathrm{Z}>0,-\infty<\propto<\infty
$$

where $\boldsymbol{\alpha}$ is a real parameter. The classical Euler-Poisson-Darboux equation is defined as [4]

$$
\frac{\partial^{2} u}{\partial r^{2}}+\frac{\alpha}{r} \frac{\partial u}{\partial r}=\sum_{i=1}^{n} \frac{\partial^{2} u}{\partial z_{i}^{2}}
$$

For equation ( 1 ), if $\mathrm{n}=1$, then this equation can be defined as

$$
\begin{gathered}
\frac{\partial^{2} u}{\partial r^{2}}+\frac{\alpha}{r} \frac{\partial u}{\partial r}=-\frac{\partial^{2} u}{\partial z^{2}} \\
\frac{\partial^{2} u}{\partial r^{2}}+\frac{\alpha}{r} \frac{\partial u}{\partial r}=\frac{\partial^{2} u}{\partial z^{2}}
\end{gathered}
$$

where $\boldsymbol{\alpha}$ is a real parameter. Equation (3) is an Elliptic Euler-Poisson-Darboux equation. It is also referred to as a generalized axisymmetric Laplace equation $[5,6]$. For $\boldsymbol{\alpha}=1$, it is the axismmetric Laplace equation, which was studied in [7]. Equation (4) is a hyperbolic Elliptic Euler-PoissonDarboux equation. The Euler-Poisson-Darboux equations was considered for the first time by Euler [8] and later by Poissn [9], Riemann [10], and Darboux [11]. In the recent time, it was studied by a number of authors $[1,4,12]$ because of its importance in mathematics and mathematical physics. 
In this article. we use the method of Lie group theory. Symmetry group methods are amongst powerful universal tools for the study of differential equations. There has been a rapid progress on these methods over the last few decades. Methods and algorithms were introduced for classifying subalgebras of Lie algebra. New results on the structure and classification of abstract finite and infinite dimensional Lie algebra were published [13]. Also, methods for solving group classification problems for differential equations greatly facilitated to systematically obtain exact analytical solutions $[13,14$, 15]. During the solution, we used some methods of solving partial differential equations and ordinary differential equations $[16,17,18]$.

Symmetry of Elliptic Euler-Poisson-Darboux Equation

Similarity is an extremely powerful method of solving the linear and nonlinear differential equations $[13,14]$. For solving the Elliptic Euler-Poisson-Darboux equation (3), we need to introduce the following Lie algebra approach as a transformation method.

We apply Lie group method to solve (3), as follows

$$
\left.X^{2}\left(\frac{\partial^{2} u}{\partial r^{2}}+\frac{\alpha}{r} \frac{\partial u}{\partial r}+\frac{\partial^{2} u}{\partial r^{2}}\right)\right|_{u_{r r}=-\frac{\alpha}{r} u_{r}-u_{r r}}=0
$$

Its $2^{\text {nd }}$ extension, $X^{2}$, is defined by

$$
X^{[2]}=X+\eta_{r}^{(1)} \frac{\partial}{\partial u_{r}}+\eta_{z}^{(1)} \frac{\partial}{\partial u_{z}}+\eta_{r r}^{(2)} \frac{\partial^{2}}{\partial u_{r r}}+\eta_{z z}^{(2)} \frac{\partial^{2}}{\partial u_{z z}}
$$

The generator $X$ is defined by

$$
X=\xi_{1} \partial r+\xi_{2} \partial z+\eta \partial u
$$

where $\eta_{r}^{(1)}, \eta_{z}^{(1)}, \eta_{r r}^{(2)}, \eta_{z z}^{(2)}$ are

$$
\begin{gathered}
\eta_{r}^{(1)}=\eta_{r}+\left[\eta_{u}-\xi_{1 r}\right] u_{r}-\xi_{2 r} u_{z}-\xi_{1 u} u_{1}^{(2)}-\xi_{2 u} u_{r} u_{z} \\
\eta_{z}^{(1)}=\eta_{z}+\left[\eta_{u}-\xi_{2 z}\right] u_{z}-\xi_{1 z} u_{r-} \xi_{2 u} u_{2}^{(2)}-\xi_{1 u} u_{r} u_{z} \\
\eta_{r r}^{(2)}=\eta_{r r}+\left[2 \eta_{r u}-\xi_{r r}\right] u_{r}-\xi_{2 r r} u_{z}+\left[\eta_{u}-2 \xi_{1 r}\right] u_{r r}-2 \xi_{2 r} u_{r z}+ \\
{\left[\eta_{u u}-2 \xi_{1 r u}\right](u)^{2}-2 \xi_{2 x 1} u_{r} u_{z 2}-\xi_{1 u u}\left(u_{r}\right)^{3}-\xi_{2 u u}\left(u_{r}\right)^{2} u_{z}-3 \xi_{i u} u_{r} u_{r r}} \\
-\xi_{2 u} u_{z} u_{r r}-2 \xi_{2 u} u_{r} u_{r z}, \\
\eta_{z z}^{(2)}=\eta_{z z}+\left[2 \eta_{z u}-\xi_{2 z z}\right] u_{z}-\xi_{1 z z} u_{r}+\left[\eta_{u}-2 \xi_{2 z}\right] u_{z z}-2 \xi_{1 z} u_{r z} \\
{\left[\eta_{u u}-2 \xi_{2 z u}\right]\left(u_{z}\right)^{2}-2 \xi_{1 z u} u r u_{z}-\xi_{2 u u}\left(u_{z}\right)^{3}-\xi_{1 u u} u_{r}\left(u_{z}\right)^{2}-3 \xi_{2 u} u_{z} u_{z z}}
\end{gathered}
$$

The invariance condition for equation (3) is

$$
\eta_{r r}-\frac{\alpha}{r^{2}} u_{r} \xi_{1}+\frac{\alpha}{r} \eta_{r}+\left.\eta_{z z}\right|_{u_{r r}=\cdots}=0
$$

By substituting Eqs. (8-11) and write equation (3) as

$$
\frac{\partial^{2} u}{\partial r^{2}}=-\frac{\alpha}{r} \frac{\partial u}{\partial r}-\frac{\partial^{2} u}{\partial z^{2}}
$$

into (12), we get

$$
\eta_{r r}+\left[2 \eta_{r u}-\xi_{r r}\right] u_{r}-\xi_{2 r r} u_{z}+\left[\eta_{u}-2 \xi_{1 r}\right] u_{r r}-2 \xi_{2 r} u_{r z}+
$$$$
\left[\eta_{u u}-2 \xi_{1 r u}\right](u r)^{2}-2 \xi_{2 x 1} u_{r} u_{z 2}-\xi_{1 u u}\left(u_{r}\right)^{3}-\xi_{2 u u}\left(u_{r}\right)^{2} u_{z}-3 \xi_{i u} u_{r} u_{r r}-\xi_{2 u} u_{z} u_{r r}-2 \xi_{2 u} u_{r} u_{r z},-
$$$$
\frac{\alpha}{r^{2}} u_{r} \xi_{1}+\frac{\alpha}{r}\left(\eta_{r}+\left[\eta_{u}-\xi_{1 r}\right] u_{r}-\xi_{2 r} u_{z}-\xi_{1 u} u_{1}{ }^{(2)}-\xi_{2} u_{r} u_{z}\right)+\eta_{z z}+\left[2 \eta_{z u}-\xi_{2 z z}\right] u_{z}-\xi_{1 z z} u_{r}+
$$$$
\left[\eta_{u}-2 \xi_{2 z}\right] u_{z z}-2 \xi_{1 z} u_{r z}\left[\eta_{u u}-2 \xi_{2 z u}\right] u_{z}^{2}-2 \xi_{1 z} u_{z} u_{r r} \xi_{2 u u} u_{z}^{2}-\xi_{1 u u} u_{r} u_{z}^{2}-3 \xi_{2 u} u_{z} u_{z z}
$$$$
-\xi_{1 u} u_{r} u_{z z}-2 \xi_{1 u} u_{z} u_{r z},=0
$$

From this equation, we obtain a polynomial equation in $u_{r}, u_{z}, u_{r r} u_{r}^{2}, u_{z}^{2}, u_{z r}, u_{z z}$,

From the coefficient of $u_{z} u_{r r}, u_{r} u_{z z}$ we have got

$$
\xi_{1 u}=\xi_{2 u}=0 \text {. }
$$

From the coefficient of $u_{z r}$, we have got

$$
\xi_{2 r}=-\xi_{1 z} \text {. }
$$

From the coefficient of $u_{z z}$, we have got

$$
\xi_{1 r}=\xi_{2 z} \text {. }
$$

From the coefficient of $u_{z}$ we have got

$$
-\xi_{2 r r}-\frac{\alpha}{r} \xi_{2 r}+\left(2 \eta_{z u}-\xi_{2 z z}\right)=0
$$


From the coefficient of $u_{r}$, we have got

$$
-\frac{\alpha}{r^{2}} \xi_{1}-\frac{\alpha}{r} \xi_{1 r}+\left(2 \eta_{r u}-\xi_{1 r r}\right)=0
$$

From the free Coefficient we have got

$$
\eta_{r r}+\frac{\alpha}{r} \eta_{r}+\eta_{z z}=0
$$

We have noticed that, due to the complexity of finding Lie group of transformation from Eq. (13) through Eqs. (14-19), so we derive eq(3) repect to $r$ to obtain the equation of the form:

$$
\frac{\partial^{3} u}{\partial r^{3}}-\frac{\alpha}{r^{2}} \frac{\partial u}{\partial r}+\frac{\alpha}{r} \frac{\partial^{2} u}{\partial r^{2}}+\frac{\partial^{3} u}{\partial z^{2} \partial_{r}}=0
$$

The third extension of generator is:

$$
X^{[3]}=X+\eta_{r}^{1} \frac{\partial}{\partial r}+\eta_{r r}^{2} \frac{\partial}{\partial u_{r r}}+\eta_{r r r}^{3} \frac{\partial}{\partial u_{r r r}}+\eta_{z z r}^{3} \frac{\partial}{\partial u_{z z r}}
$$

Now, we find the extended infinitesimal coefficient from the formula:

$$
\begin{aligned}
& \eta_{i_{1} i_{2} \ldots i_{k}}^{[3]}=D_{i k} \eta^{(k-1)}-\left(D_{i k} \xi_{j}\right) u_{i_{1} i_{2} \ldots i_{k-1} j} \\
& \text { Where } D_{i}=\frac{\partial}{\partial r_{i}}+u_{i} \frac{\partial}{\partial u}+\cdots+u_{i_{1} i_{2} \ldots i_{m}} \frac{\partial}{\partial_{i_{1} i_{2} \ldots i_{m}}}, \quad i=1,2, \ldots \mathrm{n}
\end{aligned}
$$

Then, from (21-23), we found the extended infinitesimal coefficient.

$\eta_{r r r}^{[3]}=\eta_{r r r}+\left[3 \eta_{r r u}-\xi_{1 r r r}\right] u_{r}+\left[\eta_{u u r}-2 \xi_{1 r u u}\right] u_{r}^{2}+3\left[\eta_{u r-} \xi_{1 r r}\right] u_{r r}-3 \xi_{2 r} u_{r z}-\xi_{2 r r r} u_{z}+$ $\left[\eta_{\text {uuu }}-3 \xi_{1 r u u}\right] u_{r}^{3}-3 \xi_{2 u u r} u_{z} u_{r}^{2}-3 \xi_{1 u r} u_{r} u_{z z}-3 \xi_{2 u r} u_{z} u_{r r}-4 \xi_{2 r u} u_{z r} u_{r}+3\left[\eta_{u u}-\right.$ $\left.2 \xi_{1 r u}\right] u_{r} u_{r r}-\xi_{1 \text { uиu }} u_{r}^{4}-\xi_{2 u u u} u_{r}^{3} u_{z}-6 \xi_{1 u u} u_{r}^{2} u_{r r}-\left[2 \xi_{2 u}+\xi_{2 u u}\right] u_{r}^{2} u_{r z}-3 \xi_{2 r u} u_{r} u_{r z}-$ $\xi_{2 u} u_{r r} u_{r z}-3 \xi_{2 u u} u_{r} u_{z} u_{r r}-\left[3 \xi_{1 u}+\xi_{2 u}\right] u_{r r}^{2}-\left[\eta_{u}-3 \xi_{1 r}\right] u_{r r r}-4 \xi_{1 u} u_{r} u_{r r r}-\xi_{2 u} u_{z} u_{r r r}-$ $3 \xi_{2 r} u_{z r r}-\xi_{2 u} u_{r} u_{r r z}$

$\eta_{z z r}^{[3]}=\eta_{r z z}+\left[\eta_{z z u}-\xi_{2 z z r}\right] u_{r}+\left[2 \eta_{z u r}-\xi_{1 r z z}\right] u_{z}+\left[2 \eta_{u z-} \xi_{1 z z}-2 \xi_{2 r z u}\right] u_{r z}-\xi_{2 z z} u_{r r}+$ $\left[\eta_{u r}-2 \xi_{1 r z}\right] u_{z z}-2 \xi_{2 z u u} u_{z} u_{r}^{2}-3 \xi_{1 u} u_{z} u_{z z}-3 \xi_{2 u r} u_{z} u_{r r}+\left[\eta_{u u z}-2 \xi_{1 r z u}\right] u_{z}^{2}-\xi_{1 z} u_{z z z} u_{z}-$ $\xi_{1 u u u} u_{z}^{3}-\xi_{1 z u} u_{z} u_{r r}-\left[\eta_{r u u-} 2 \xi_{1 u z u}-\xi_{2 u u u}\right] u_{z}^{2} u_{z r}-3 \xi_{2 r u} u_{r} u_{r z}-\left[2 \eta_{u u z}-2 \xi_{2 z r u}-\xi_{1 z z u}-\right.$ $\left.\xi_{2 z u u}\right] u_{r} u_{z}-2 \xi_{1 r} u_{z z z}+\left[\eta_{u}-\xi_{1 z+} \xi_{2 z}+\xi_{2 r}\right] u_{z z r}-\left[3 \xi_{2 u}-2 \xi_{1 u}\right] u_{z} u_{z z r}-\xi_{2 u r} u_{z} u_{r z}-$ $3 \xi_{1 u u} u_{r} u_{z} u_{z z}-\left[\eta_{u u-} \xi_{2 u u}-2 \xi_{1 u z}-\xi_{2 u r}\right] u_{z z} u_{r}-\left[\eta_{u r}-\xi_{1 r z}\right] u_{z z}-2 \xi_{2 u u} u_{r} u_{z} u_{r z}-$ $\xi_{2 u u} u_{z}^{2} u_{r r}-3 \xi_{1 u u} u_{z}^{2} u_{z r}-3 \xi_{1 u} u_{z z} u_{r z}-3 \xi_{1 u r} u_{z z} u_{z}$

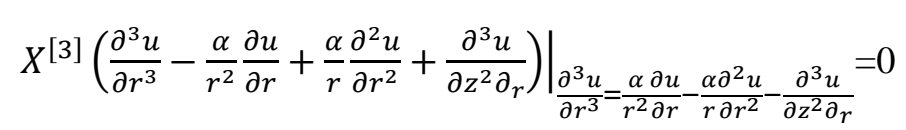

The invariance condition for equation (20) is

$$
\left.\left(\eta_{r r r}+\frac{2 \alpha}{r^{3}} \xi \frac{\partial u}{\partial r}-\frac{\alpha}{r^{2}} \eta_{r}+\frac{\alpha}{r} \eta_{r r}-\frac{\alpha}{r^{2}} \xi \frac{\partial^{2} u}{\partial r^{2}}+\eta_{z z r}\right)\right|_{\frac{\partial^{3} u}{\partial r^{3}}=\frac{\alpha \partial u}{r^{2} \partial r}-\frac{\alpha \partial^{2} u}{r \partial r^{2}}-\frac{\partial^{3} u}{\partial z^{2} \partial_{r}}}=0
$$

By the substitution of (8-11 and 24-25) into (27), we get

$\eta_{r r r}+\left[3 \eta_{r r u}-\xi_{1 r r r}\right] u_{r}+\left[\eta_{u u r}-2 \xi_{1 r u u}\right] u_{r}^{2}+3\left[\eta_{u r-} \xi_{1 r r}\right] u_{r r}-3 \xi_{2 r} u_{r z}-\xi_{2 r r r} u_{z}+$ $\left[\eta_{\text {uuu }}-3 \xi_{1 r u u}\right] u_{r}^{3}-3 \xi_{2 u u r} u_{z} u_{r}^{2}-3 \xi_{1 u r} u_{r} u_{z z}-3 \xi_{2 u r} u_{z} u_{r r}-4 \xi_{2 r u} u_{z r} u_{r}+3\left[\eta_{u u}-\right.$ $\left.2 \xi_{1 r u}\right] u_{r} u_{r r}-\xi_{1 u u u} u_{r}^{4}-\xi_{2 u u u} u_{r}^{3} u_{z}-6 \xi_{1 u u} u_{r}^{2} u_{r r}-\left[2 \xi_{2 u}+\xi_{2 u u}\right] u_{r}^{2} u_{r z}-3 \xi_{2 r u} u_{r} u_{r z}-$ $\xi_{2 u} u_{r r} u_{r z}-3 \xi_{2 u u} u_{r} u_{z} u_{r r}-\left[3 \xi_{1 u}+\xi_{2 u}\right] u_{r r}^{2}+\left[\eta_{u}-3 \xi_{1 r}\right] u_{r r r}-4 \xi_{1 u} u_{r} u_{r r r}-\xi_{2 u} u_{z} u_{r r r}-$ $3 \xi_{2 r} u_{z r r}-\xi_{2 u} u_{r} u_{r r z}+2 \frac{\alpha}{r^{3}} \frac{\partial u}{\partial r} \xi_{1}-\frac{\alpha}{r^{2}}\left(\eta_{r}+\left[\eta_{u}-\xi_{1 r}\right] u_{r}-\xi_{2 r} u_{z} \xi_{1 u} u_{1}^{2}-\xi_{2} u_{r} u_{z}\right)+\frac{\alpha}{r}\left(\eta_{r r}+\right.$ $\left[2 \eta_{r u}-\xi_{r r}\right] u_{r}-\xi_{2 r r} u_{z}+\left[\eta_{u}-2 \xi_{1 r}\right] u_{r r}-2 \xi_{2 r} u_{r z}-\xi_{2 u} u_{z} u_{r r}-2 \xi_{2 u} u_{r} u_{r z}-$

$\left.\left[\eta_{u u}-2 \xi_{1 r u}\right](u)^{2}-2 \xi_{2 x 1} u_{r} u_{z 2}-\xi_{1 u u}\left(u_{r}\right)^{3}-\xi_{2 u u}\left(u_{r}\right)^{2} u_{z}-3 \xi_{i u} u_{r} u_{r r}\right)-\frac{\alpha}{r^{2}} \frac{\partial^{2} u}{\partial r^{2}} \xi_{1}+\left(\eta_{r z z}+\right.$ $\left[\eta_{z z u}-\xi_{2 z z r}\right] u_{r}+\left[2 \eta_{z u r}-\xi_{1 r z z}\right] u_{z}+\left[2 \eta_{u z-} \xi_{1 z z}-\left[2 \eta_{u z-} \xi_{1 z z}-2 \xi_{2 r z u}\right] u_{r z}-\xi_{2 z z} u_{r r}+\right.$ $\left[\eta_{u r}-2 \xi_{1 r z}\right] u_{z z}-2 \xi_{2 z u u} u_{z} u_{r}^{2}-3 \xi_{1 u} u_{z} u_{z z}-3 \xi_{2 u r} u_{z} u_{r r}+\left[\eta_{u u z}-2 \xi_{1 r z u}\right] u_{z}^{2}-\xi_{1 z} u_{z z z} u_{z}-$ $\xi_{1 u и u} u_{z}^{3}-\xi_{1 z u} u_{z} u_{r r}-\left[\eta_{\text {ruu }} 2 \xi_{1 u z u}-\xi_{2 u u u}\right] u_{z}^{2} u_{z r}-3 \xi_{2 r u}-\left[2 \eta_{u u z}-2 \xi_{2 z r u}-\xi_{1 z z u}-\right.$ $\left.\xi_{2 z u u}\right] u_{r} u_{z}-2 \xi_{1 r} u_{z z z}+\left[\eta_{u}-\xi_{1 z+} \xi_{2 z}+\xi_{2 r}\right] u_{z z r}-\left[3 \xi_{2 u}-2 \xi_{1 u}\right] u_{z} u_{z z r}-\xi_{2 u r} u_{z} u_{r z}-$ $3 \xi_{1 u u} u_{r} u_{z} u_{z z}-\left[\eta_{u u-} \xi_{2 u u}-2 \xi_{1 u z}-\xi_{2 u r}\right] u_{z z} u_{r}-\left[\eta_{u r}-\xi_{1 r z}\right] u_{z z}-2 \xi_{2 u u} u_{r} u_{z} u_{r z}-$ $\left.\xi_{2 u u} u_{z}^{2} u_{r r}-3 \xi_{1 u u} u_{z}^{2} u_{z r}-3 \xi_{1 u} u_{z z} u_{r z}-3 \xi_{1 u r} u_{z z} u_{z}\right)$.

From this equation, we obtain a polynomial equation in $u_{r}, u_{z}, u_{r r z}, u_{r}^{3}, u_{z}^{3}, u_{z r} u_{z z z}, u_{z z r}, u_{r r}, u_{z z z} u_{z r}, u_{r} u_{z}$.

The coefficient of $u_{z z} u_{z r}, u_{r r} u_{z r}$ is 


$$
\xi_{1 u}=\xi_{2 u}=0
$$

The coefficient of $u_{r z z}$ is

$$
\eta_{u-} \xi_{1 r}+\xi_{2 z}-2 \xi_{2 r}=0
$$

The coefficient of $u_{z z z}$ is

The coefficient of $u_{r r z}$ is

$$
\xi_{1 r}=0
$$

The coefficient of $u_{z z}$ is

$$
\xi_{2 r}=0
$$

The coefficient of $u_{r}^{3}, u_{z}^{3}$ is

$$
\eta_{u r}=0
$$

The coefficient of $u_{z r}$ is

$$
\eta_{u u u}=0
$$

The coefficient of $u_{z z r}$ is

$$
2 \eta_{z u}-\xi_{1 z z}=0
$$

The free coefficient is

$$
\xi_{2 z}-\xi_{1 z}=0 \rightarrow \xi_{2 z}=\xi_{1 z}
$$

$$
\eta_{r r r}-\frac{\alpha}{r^{2}} \eta_{r}+\frac{\alpha}{r} \eta_{r r}+\eta_{z z r}=0
$$

From 12, 13, 14 , 27, and 29, we have

$$
\xi_{2 z}=\xi_{1 z}=\xi_{1 u}=\xi_{2 u=} \xi_{2 r=} \xi_{1 r}=0
$$

This yields that $\xi_{1}=\xi_{2}=$ constants

$$
\text { Let } \xi_{1}=c_{1} \text { and } \xi_{2}=c_{2}
$$

of the coefficient of $u_{r r}$ is

$$
\eta_{u}=0
$$

From (33 and 35), we get
\[ \eta_{u z}=\eta_{u r}=0 \]

Then by solving this equation, we have

$$
\eta(r, z)=F(r, z) r+c_{3}
$$

or

$$
\eta(r, z)=G(r, z) z+c_{4}
$$

Since $42=43$, then we get

$$
\begin{gathered}
F(r, z)=\frac{1}{r}, \text { or } G(r, z)=\frac{1}{z} \text { and } c_{3}=c_{4}=\mathrm{c} \\
\text { Hence } \eta(r, z)=1+c
\end{gathered}
$$

Using lagrange method, we have

$$
\begin{aligned}
& \frac{\partial r}{\xi_{1}}=\frac{\partial z}{\xi_{2}}=\frac{\partial u}{\eta} \\
& \frac{\partial r}{c_{1}}=\frac{\partial z}{c_{2}}=\frac{\partial u}{1+c}
\end{aligned}
$$

This yields two independent solutions, as follows:

$$
\begin{aligned}
\frac{\partial r}{c_{1}} & =\frac{\partial z}{c_{2}} \longrightarrow \frac{1}{c_{1}} r-\frac{1}{c_{2}} z=S \\
\frac{\partial z}{c_{2}} & =\frac{\partial u}{1+c} \longrightarrow \frac{1}{1+c} u=\frac{1}{c_{2}} z+f(S) \\
u & =\frac{(1+c)}{c_{2}} z+(1+c) f(S)
\end{aligned}
$$

To find $f(S)$, we substitute (49) in (3), as follows:

$$
\begin{aligned}
& u_{r}=\frac{1}{c_{1}}(1+c) f^{\prime}(S) \\
& u_{r r}=\frac{1}{c_{1}^{2}}(1+c) f^{\prime \prime}(S) \\
& u_{z z}=\frac{1}{c_{2}^{2}}(1+c) f^{\prime \prime}(S)
\end{aligned}
$$

Substituting (50-52) in (3) yields the ordinary differential equation $\frac{1}{c_{1}^{2}}(1+c) f^{\prime \prime}(S)+\frac{\alpha}{r} \frac{1}{c_{1}}(1+c) f^{\prime}(S)+\frac{1}{c_{2}^{2}}(1+c) f^{\prime \prime}(S)=0$ 


$$
f^{\prime \prime}(S)+\frac{\alpha}{r} c_{1} f^{\prime}(S)+\frac{c_{1}^{2}}{c_{2}^{2}} f^{\prime \prime}(S)=0
$$

We have got

$$
f(S)=A+B e^{-\frac{\alpha c_{2}^{2}}{r\left(c_{1}^{2}+c_{2}^{2}\right)} S}
$$

By substituting (54) in (49), we get

$$
u=\frac{(1+c)}{c_{2}} z+(1+c)\left(A+B e^{-\frac{\alpha c_{2}^{2}}{r\left(c_{1}^{2}+c_{2}^{2}\right)} S}\right)
$$

\section{Acknowledgments}

I would like to thank Mustansiriyah University (www.uomustansiriyah.edu.iq) Baghdad-Iraq for its support in the present work.

\section{References}

1. Kipriyanov. I. A.1997. Singular Elliptic Boundary Value problems, Nauka, Moscow,. (in Russian).

2. Weinstein. A. 1952. Sur le probl'eme de Cauchy pour l equation de Poisson et l'equation des ondes, C. R. Acad. Sci. Paris, 234 , 2584-2585.

3. Weinstein. A . 1954. On the wave equation and the equation of Euler-Poisson, Proceedings of Symposia in Applied Mathematics, Wave motion and vibration theory, Mcgraw-Hill Book company, New York-Toronto-London, 137-147

4. Shishkina. E. L. and Sitnik. S. M. 2017. General form of the Euler-Poisson-Darboux Equation and application of the transmutation method, Electronic Journal of Differential Equations, 2017(177): $1-20$.

5. Weinstein, A. 1948. Trans. Amer. Math. Soc., 63(2): 342-354.

6. Weinstein. A. 1955. The generalized radiation problem and the Euler-Poisson-Darboux equation, summa Brasiliensis Mathematica's , 3(1955): 125-147.

7. Beltrami, E., Men. R. 1880. Accad. Sci. Bologna, 2: 461-505 (Opere mat., 1911, 3: 349-382).

8. Euler, L. 1914. Institutiones calculi integralis, 3, petropoli, 1770, pt. 2, chaps. 3, 4,5 (Opera Omnia, Leipzig, 1914, ser. 1, vol. pp.212-230).

9. Poisson, S.D. 1823. J. de L'Ecole Polytechnique, 1823, ser. 19: 215-248.

10. Riemann, B. 1948. in Sochineniya (Works), Moscow, 1948, pp. 376-395.

11. Darboux, G.1915. Leçons sur la théorie générale des surfaces et les applications géométriques du calcul infinitesimal, Paris, 2 ed., 1915 (1 ed., 1888), vol. 1.

12. Shishkina. E. L. 2018. Generalized Euler-Poisson-Darboux equation and singular Klein-Gordon equation, Journal of Physics: Conf. Series 973 (2018): 012058

13. Bluman. G. W., and Kumei. S. 1989. Symmetries and differential equation, New York Berlin Heidelbrg.

14. Jasim. M. J and John. Z. 2015. Some new similarity of Einstein field equation for spherical symmetric metric of class two, Journal of Applied Mathematics and Computation, 253(2015).

15. Aldhlki T. Jassim, 2020. Nonclassical Symmetry of Differential Equations. Iraqi Journal of Science. (Special Issue) The first Conference of Mathematics-2020 (Jassim, 2020)

16. John. W. C., David. G. S. 2016. Ordinary Differential Equations: Basics and Beyond; springer Science+Business Media New York (2016)

17. Nandakumaran. A. K., Datti. P. S and George. R. K. 2017. Ordinary Differential Equations: Principles and Applications ;Cambridge University Press 2017

18. Rasheed M. Aand Chlebik M. 2020. Blow-up Rate Estimates and Blow -up Set for a System of two Heat Equations with Coupled Nonlinear Nwumann Boundary conditions. Iraqi Journal of Scince. 61(1): 147-152. (M., 2020) 